\title{
Finger nose proprioception test (case study)
}

\author{
Author: Khin Bo
}

\section{Introduction}

The author published on the finger nose proprioceptive test (FNPT) in 2017. ${ }^{1}$ FNPT is a bedside test to see if patient can touch his/her nose with his/her finger when the eyes are closed (Fig 1a). Patients with proprioceptive impairment will miss the tip of the nose (Fig 1b). As an extension of that research, can proprioception be improved by enhancing the remaining sensory pathways (eg temperature, pain, vibration)?

\section{Materials and methods}

A 30-year-old patient with generalised severe proprioceptive impairment from axonal ataxic polyneuropathy (variant of Guillain Barre syndrome). FNPT was severely positive in the left hand. He could still feel temperature reasonably well. Left fingers were immersed in ice cubes until the patient reported feeling very cold in the fingers. FNPT was repeated.

\section{Results and discussion}

There was about $75 \%$ improvement in locating the tip of the nose with the eyes closed (Fig 1c).

\section{Conclusion}

Impairment in proprioception can be improved by enhancing the remaining sensory pathway (emperature sensation in this case).

This is just a case report and further studies need to validate the finding.

\section{Conflict of interest statement}

None declared.

\section{Reference}

1 Bo K. Finger nose proprioceptive test. J Neuroinfect Dis 2017:8:265.
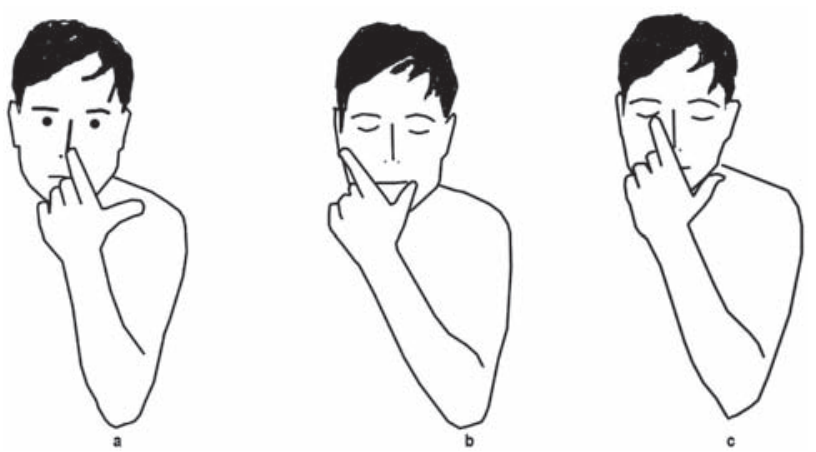

Fig 1. The test and result
Author: Rehab Medicine Service, Northern Lincolnshire \& Goole NHS Foundation Trust, UK 